\title{
Relationship Between Thyroid Cancer and Hashimoto's Disease
}

\section{Relación entre Neoplasia de Tiroides y Tiroidi- tis de Hashimoto}

XVIII International Seminar on Health, Food and Human Nutrition

Corresponding Author:

Dr. Marcelo Ramiro Montúfar Silva

dr_marmontufar@yahoo.com

Published: 9 September 2021

Production and Hosting by

Knowledge E

(c) Dr. Marcelo Ramiro Montúfar Silva et al. This article is distributed under the terms of the Creative Commons Attribution License, which permits unrestricted use and redistribution provided that the original author and source are credited.
Dr. Marcelo Ramiro Montúfar Silva, Dra. Jessica Paola Cevallos Montalvo ${ }^{2}$, Dr. Byron Mauricio Sánchez Andino ${ }^{3}$, Dra. María Johanna Jima Sanchez ${ }^{3}$, and Dra. Cecilia Alejandra García Ríos ${ }^{4}$

${ }^{1}$ Docente ESPOCH Médico Internista, Riobamba, Ecuador

${ }^{2}$ Medico Posgradista de Dermatología, Riobamba, Ecuador

${ }^{3}$ Médico Internista

${ }^{4}$ Docente ESPOCH, Pediatra, Riobamba, Ecuador

\section{Abstract}

Introduction: Globally, Ecuador ranks fifth among the countries with the highest incidence of thyroid carcinoma in women, below Korea, Brazil, Italy and New Celedonia. The aim of this research was to mention the relationship between thyroid cancer and Hashimoto's thyroiditis at Pablo Arturo Suárez Hospital in Quito in the years 2014 to 2017. Methodology: A cross sectional study was conducted. The data were taken from the clinical records in the statistical service, 105 cases of thyroid cancer were found. In the laboratory results 21 patients had anti TPO and/or anti TG positive antibodies. Conclusion: $20 \%$ of patients with thyroid cancer had a history of Hashimoto's thyroiditis.

Keywords: thyroid cancer, Hashimoto's thyroiditis, autoimmune thyroiditis.

\section{Resumen}

Introducción: A nivel mundial el Ecuador ocupa el quinto lugar entre los países con mayor incidencia de carcinoma de tiroides en mujeres, por debajo de Corea, Brasil, Italia y Nueva Celedonia. Objetivo: El objetivo de la presente investigación fue mencionar la relación entre cáncer de tiroides y tiroiditis de Hashimoto en el Hospital Pablo Arturo Suárez de la ciudad de Quito en los años 2014 a 2017. Metodología: se desarrolló un estudio de tipo transversal los datos se tomaron de los expedientes clínicos en el servicio de estadística, donde se encontró 105 casos de estudios histopatológicos compatibles con neoplasia tiroidea. Resultados: En los resultados de laboratorio se evidenció que 21 pacientes tuvieron anticuerpos anti TPO y/o anti TG positivos. Conclusión: El 20\% de pacientes con cáncer de tiroides tuvieron antecedente de enfermedad autoinmune tiroidea.

Palabras Clave: cáncer de tiroides, tiroiditis de Hashimoto, tiroiditis autoinmune. 


\section{Introducción}

Dentro de los tumores malignos de la glándula tiroidea encontramos a los adenocarcinomas de tipo diferenciados tanto papilar como folicular, carcinomas no diferenciados, carcinoma medular, y en menos frecuencia linfomas, enfermedad metastásica entre otras [1].

La tasa de cáncer tiroideo papilar es más alta en pacientes asiáticas (10.96 por 100000 mujeres por año) y más baja en mujeres de raza negra (4 por 100000 mujeres por año), la tasa de cáncer folicular no varía por raza o etnia y el cáncer medular es más alto en mujeres de origen hispano (0,21 por 100.000 mujeres/año) [2].

En hombres la tasa de cáncer tiroideo papilar y folicular son altas en raza blanca (3,8 y 5,8 por 100.000 hombres/año respectivamente), el cáncer medular es más alto en hispanos (0,18 por 100.000 hombres/año) y el cáncer anaplásico es más alto en hombres de procedencia asiática (0,11 por 100.000 hombres año) [2].

La tiroiditis de Hashimoto es una enfermedad autoinmune en donde las células son destruidas por un proceso inmune mediado por anticuerpos provocando hipotiroidismo subclínico o manifiesto, es la causa más común de hipotiroidismo en países desarrollados, en países subdesarrollados aún se toma en cuenta como etiología la falta de yodo. Es mayor en el sexo femenino: 3,5 por 1000 mujeres cada año y 0,8 por 1000 hombres cada año [3].

Hay varios estudios publicados que demuestran la asociación entre tiroiditis de Hashimoto y cáncer de tiroides sobre todo el tipo papilar. Fulciniti F. [4] menciona que el carcinoma tiroideo folicular en su variante sólida, es visto más frecuentemente en paciente pediátricos asiáticos con o sin historia previa de irradiación en región cervical.

En su investigación Leni et al. [5] encontraron que la tiroiditis estaba presente en el 33,3\% de pacientes con cáncer tiroideo papilar, concluyendo en este estudio que uno de cada tres pacientes con cáncer tiroideo papilar tienen el antecedente de Hashimoto.

En el estudio realizado por Slijepcevic et al. [6], el 22,7\% de los pacientes con microcarcinoma tiroideo papilar tenia tiroiditis de Hashimoto: Con un OR 1,54, 95\% $\mathrm{Cl} 1,15-2,05, p<0,003$. Siendo estadísticamente significativa la asociación de las dos patologías. Akin et al. [7], demuestra en su estudio retrospectivo que de 141 pacientes con microcarcinoma papilar 26,2\% tenían tiroiditis de Hashimoto.

La investigación planteada por Baser et al. [8] analizaron retrospectivamente 919 pacientes con carcinoma papilar tiroideo y encontraron que 317 de los participantes $(34,5 \%)$ tuvieron Tiroiditis de Hashimoto. Finalmente, en un estudio retrospectivo realizado en el 2016 por Lai et al. [9] se demostró que de los pacientes con microcarcinoma papilar tiroideo el $26 \%$ tuvo tiroiditis de Hashimoto.

Con estos antecedentes podemos observar que existe una asociación entre la tiroiditis de Hashimoto y el cáncer tiroideo. Se ha descrito que la presencia de tiroiditis crónica en pacientes con carcinoma papilar muestra un impacto positivo en el curso de la enfermedad, relacionado principalmente con menos crecimiento del tumor primario, menor invasión y menos metástasis [10]. 
Existe evidencia que la tiroiditis de Hashimoto incrementa el riesgo de Cáncer tiroideo diferenciado en pacientes con valores de hormonas tiroideas dentro de parámetros o levemente hipotiroideos, no siendo así en pacientes con tetrayodotironina moderada o severamente alta [11].

Se ha propuesto que los mecanismos que aclaran el vínculo entre la tiroiditis de Hashimoto y el microcarcinoma papilar son: Estimulación con TSH (tirotropina), expresión de ciertos protooncogenes, presencia de quimiocinas y otras moléculas que son producidas por el infiltrado linfocítico [12, 13].

Así pues los cuatro mecanismos patógenos que relacionan la asociación de tiroiditis de Hashimoto y cáncer tiroideo son la autoinmunidad preexistente que conduce a neoplasias malignas debido a la reacción inflamatoria, inmunidad contra células tumorales preexistentes que conducen a enfermedades específicas autoinmunes, tolerancia del sistema inmune que conduce a malignidad a pesar de la autoinmunidad que tiene el paciente y los niveles de especies reactivas de oxígeno pueden aumentar en pacientes con carcinoma tiroideo y tiroiditis [14-19].

La bibliografía menciona investigaciones desarrolladas en otros países, por lo que es importante realizar estudios en nuestro medio para analizar el comportamiento de la tiroiditis de Hashimoto y neoplasia tiroidea, las mimas que tienen un alto índice de presentación sobre todo en zonas pertenecientes a la sierra central, investigación cuyo objetivo es mencionar la relación entre tiroiditis de Hashimoto y carcinoma de tiroides en el Hospital Pablo Arturo Suárez de Quito en los años 2014 a 2017.

\section{Métodos}

Se planteó un estudio descriptivo observacional transversal ya que se examinó la presencia o ausencia de una enfermedad (cáncer de tiroides) u otro resultado de interés, en relación con la existencia o no de una exposición (enfermedad autoinmune tiroidea), ambos hechos ocurriendo en población específica durante un tiempo determinado [20-22].

Se recolecto los datos de los expedientes clínicos de todos los pacientes que con diagnóstico de cáncer de tiroides por histopatología del Hospital Pablo Arturo Suárez en los años 2014 a 2017, los criterios de inclusión y exclusión se especifican en la Tabla 1.

La estadística se hizo en base a mediciones numéricas y recolección de datos, con su respectivo análisis que permitió establecer la relación de las patologías de estudió. La información obtenida durante el estudio se tabulo a través del programa Excel y programa SPSS versión 24.0.

\section{Resultados}

Al analizar los datos de 105 historias clínicas (Tabla 2), que cumplieron con los criterios de inclusión y exclusión (diagnóstico de cáncer de tiroides y resultado de laboratorio de anticuerpos antiperoxidasa y anti tiroglobulina), de los expedientes clínicos que 
reposan en el servicio de estadística, se encontró que 21 pacientes (20\%), tuvieron el antecedente de tiroiditis de Hashimoto.

\section{Table 1}

Criterios de inclusión y exclusión: La tabla muestra los resultados de inclusión y exclusión del estudio.

\section{Criterio de inclusión \\ Pacientes con diagnóstico de neoplasia de tiroides que acudieron al hospital Pablo Arturo Suárez de la ciudad de Quito entre los años 2014 y 2017. \\ Criterios de exclusión \\ Pacientes que no tengan resultado de Anti TPO (peroxidasa) y Anti TG (tiroglobulina), antes del diagnóstico de neoplasia tiroidea.}

Elaborado por: Sánchez B. (2019).

\section{Table 2}

Relación entre cáncer de tiroides y tiroditis de hashimoto: La tabla muestra los resultados de pacientes con tiroiditis de Hashimoto previo el diagnóstico de cáncer de tiroides.

\begin{tabular}{l|ll} 
Tiroiditis de hashimoto & Frecuencia & Porcentaje \\
SI & 21 & 20,0 \\
NO & 84 & 80,0 \\
Total & 105 & 100,0
\end{tabular}

Fuente: Historias Clínicas del Hospital Provincial General Pablo Arturo Suárez de enero 2014 a diciembre 2017.

Elaborado por: Sánchez B. (2019).

\section{Discusión}

En la presente investigación se observó que un $20 \%$ de pacientes con cáncer de tiroides tuvieron el antecedente clínico de tiroiditis de Hashimoto, estos datos concuerdan con literatura de Colombia, Brasil, Argentina, España, Italia y Estados Unidos [23-25], donde se menciona que existe relación entre la neoplasia tiroidea y autoinmunidad.

Priscila Carneiro y su grupo de estudio en Brasil encontraron prevalencia de nódulos tiroideos en pacientes con tiroiditis de Hashimoto en el 50,7\% (39/77) y la de carcinoma tiroideo del 7,8\% (6/77) de los casos [26].

En el departamento de endocrinología de la universidad de Barcelona, en el Hospital Universi $\neg$ tario de Bellvitgede España, José Manuel Gómez ha desarrollado varias investigaciones donde relacionan estas dos enfermedades e indica la enfermedad tiroidea autoinmune como posible precursor de neoplasia tiroidea [27].

Varios estudios realizados en los estados unidos describen la relación existente entre estas dos patologías, Penta L, menciona antecedente de tiroiditis en pacientes con neoplasia tiroidea en un 23\% [28] y Hershman JM en $14 \%$ de los 753 pacientes incluidos en su análisis [29].

Cipolla C, encontró antecedente de tiroiditis en 19 de 71 pacientes con cáncer de tiroides $(26,7 \%$ ) y en 8 pacientes con bocio $(8,9 \%)$, lo que fue una diferencia significativa 
( $p<0,02$ ). Trece de los pacientes con Hashimoto, en su mayoría con forma nodular, presentaron neoplasia papilar (27,6\%). Las dos enfermedades coexistieron en varios aspectos morfológicos, inmunohistoquímicos y biomoleculares, concluyendo que el aumento de la incidencia de carcinoma papilar en pacientes con tiroiditis podría indicar esta enfermedad como precursora de cáncer de tiroides [30].

Los mecanismos relacionados con la presentación conjunta de estas dos patologías aún no están descritos en un $100 \%$ sin embargo existen varias teorías como la autoinmunidad preexistente que conduce a carcinomas por reacción inflamatoria, inmunidad contra células tumorales preexistentes, tolerancia del sistema inmune y alteración en los niveles de especies reactivas de oxígeno, como se mencionó en párrafos anteriores.

\section{Conclusiones}

- La presente investigación mostró que los datos sobre tiroiditis autoinmune y cáncer de tiroides en la ciudad de Quito son similares a las descritas en diferentes estudios a nivel mundial.

- En los resultados de laboratorio se evidenció que 20\% de los pacientes tuvieron anticuerpos anti peroxidasa y/o anti tiroglobulina positivos en relación con tiroiditis de Hashimoto previo el diagnóstico de neoplasia de tiroides.

\section{Limitaciones}

El hospital donde se realizó la investigación es una unidad de segundo nivel por lo que se limitó el tamaño de la muestra.

\section{Recomendaciones y Fortalezas}

- Son necesarios más estudios para analizar el comportamiento de estas dos variables en nuestro medio.

- Realizar investigaciones de tipo prospectivas sobre los factores relacionados con el cáncer de tiroides como: Niveles de hormonas tiroideas, consumo de dieta bociógena y lugar de residencia.

\section{Declaración de Responsabilidad}

Todos los puntos de vista expresados son de su entera responsabilidad de los autores y no de la Escuela Superior Politécnica de Chimborazo.

\section{Fuentes de Apoyo}

Servicio de estadística Hospital Pablo Arturo Suárez. 


\section{Conflicto de Interés}

Ninguno.

\section{References}

[1] Gardner D, Shobck D. Greenspan's basic and clinical endocrinology. $9^{\text {th }}$ ed. Mexico: McGraw-Hill Companies; 2011. p. 217-226.

[2] Aschebrook-Kilfoy B, Ward MH, Sabra MM, Devesa SS. Thyroid cancer incidence patterns in the United States by histologic type, 1992-2006. Thyroid. 2011;21(2):125-134.

[3] Mincer DL, Jialal I. Hashimoto Thyroiditis. StatPearls;2019. Available from: https://www.ncbi.nlm.nih.gov/ books/NBK459262/

[4] Fulciniti F, Barizzi J, Trimboli P, Giovanella L. Solid papillary thyroid carcinoma with Hashimoto's thyroiditis: description of a further case with challenging cytological features. BMJ Case Rep. 2019;12(1).

[5] Leni A, Vita R, Magliolo E, et al. One-third of an archivial series of papillary thyroid cancer (years 2007-2015) has coexistent chronic lymphocytic thyroiditis, which is associated with a more favorable tumor-node-metastasis staging. Front Endocrinol. 2017;8(337):1-7.

[6] Slijepcevic N, Zivaljevic V, Marinkovic J, Sipetic S, Diklic A, Paunovic I. Retrospective evaluation of the incidental finding of 403 papillary thyroid microcarcinomas in 2466 patients undergoing thyroid surgery for presumed benign thyroid disease. BMC Cancer. 2015;15(330).

[7] Akin S, Yazgan AD, Akin S, Kilic M, Yetisir F, Bayraktar M. Prediction of central lymph node metastasis in patients with thyroid papillary microcarcinoma. Turk J Med Sci. 2017;47(6):1723-7.

[8] Baser H, Ozdemir D, Cuhaci N, et al. Hashimoto's thyroiditis does not affect ultrasonographical, cytological, and histopathological features in patients with papillary thyroid carcinoma. Endocr Pathol. 2015;26(4):356-64.

[9] Lai X, Zhang B, Jiang $Y$, et al. Sonographic and clinical features of papillary thyroid microcarcinoma less than or equal to five millimeters: a retrospective study. PloS One. 2016;11(2).

[10] Myshunina T, Guda B, Bolgov M, Mikhailenko N, Tronko N. Differentiated thyroid carcinomas associated with chronic thyroiditis: biological and clinical properties. Exp Oncol. 2018;40(2):128-31.

[11] Paparodis R, Imam S, Todorova-Koteva K, Satii A, Jaume J. Hashimoto's Thyroiditis pathology and risk for thyroid cancer. Thyroid. 2014;24(7):1107-14.

[12] Girardi FM, Barra MB, Zettler CG. Papillary thyroid carcinoma: Does the association with Hashimoto's thyroiditis affect the clinicopathological characteristics of the disease. Braz J Otorhinolaryngol. 2015;81(3):283-7.

[13] Vita R, Leni A, Tuccari G, Benvenga S. The increasing prevalence of chronic lymphocytic thyroiditis in papillary microcarcinoma. Rev Endocr Metab Disord. 2018;19(4):301-9.

[14] Ehlers M, Schott M. Hashimoto's thyroiditis and papillary thyroid cancer: are they immunologically linked? Trends Endocrinol Metab. 2014;25(12):656-64.

[15] lb L, Salas VM. Actualización de patología tiroidea 2017. AePap; 2017. p. 161-74.

[16] Es QUÉ, Glándula LA, Son C, Del LOSS, Tiroides PDE. Linfoma primario de la tiroides linfoma primario de la tiroides. 2018.

[17] Yi JW, Park JY, Sung JY, et al. Genomic evidence of reactive oxygen species elevation in papillary thyroid carcinoma with Hashimoto thyroiditis. Endocr J. 2015;62(10):857-77.

[18] Félix J, Ramos R. Risk factors for thyroid diseases: Ambato Social Security Hospital in Ecuador. Revista Ciencias médicas Pinar del Rio. 2016;20(5):628-38.

[19] Piraino PN, Sepúlveda AN, Cavada GC. Tiroiditis crónica de Hashimoto. serie clínica. Rev Med Chil. 2010;138(7):827- 31.

[20] Álvarez G, Delgado J. Diseno de Estudios Epidemiológicos. I. El estudio transversal: Tomadondo una fotografía de la salud y la enfermedad. Bol Clin Hosp Infant Endo Son. 2015;32(1):26-34.

[21] Rodríguez M, Mendivelso F. Diseño de investigación de Corte Transversal. Rev Médica Sanitas. 2018;21(3):141-6.

[22] Manterola C, Otzen T. The most commonly used designs in clinical research. Int J Morphol. 2014;32(2):634-45.

[23] Gutiérrez-Pérez RB, Salgado ML. Evaluación de la disfunción tiroidea en estudiantes de una institución universitaria Evaluation of thyroid dysfunction in university students of an institution. Rev Salud Pública. 2016;18(6):926-34.

[24] Akdi A. Susceptibilidad genética al cáncer de tiroides: Estudios de asociación de las regiones de genoma 1p12 Y 8q. Bellaterra: Universidad Autónoma de Barcelona; 2011. 23 p. 
[25] Casos GSDE. By gammagraphy. Series of cases introducción. 2010; 18(40):235-40.

[26] Lima PCM, Moura NA, Tambascia MA, Zantut Wittmann DE. Risk factors associated with benign and malignant thyroid nodules in autoimmune thyroid diseases. ISRN Endocrinol. 2013;2013(673146):1-7.

[27] Sáez MGJ. Hashimoto's Thyroiditis and thyroid cancer. Hum Endocrinol. 2017; 1(1):1-4.

[28] Penta L, Cofini M, Lanciotti L, Leonardi A, Principi N, Esposito S. Hashimoto's disease and thyroid cancer in children: Are they associated? Front Endocrinol (Lausanne). 2018;9(565):1-5.

[29] Hershman JM. Patients with differentiated thyroid cancer and coexistent hashimoto's thyroiditis have a better prognosis than those without thyroiditis. Clin Thyroidol. 2013;25(10):225-6.

[30] Cipolla C, Sandonato L, Graceffa G, et al. Hashimoto thyroiditis coexistent with papillary thyroid carcinoma. Am Surg. 2005;71(10):874-8. 